\title{
Nota
}

\section{MÉTODOS DE QUANTIFICAÇÃO DE CARBONO E MATÉRIA ORGÂNICA EM RESÍDUOS ORGÂNICOS ${ }^{(1)}$}

\author{
Davi Lopes do $\operatorname{Carmo}^{(2)}$ \& Carlos Alberto Silva ${ }^{(3)}$
}

\begin{abstract}
RESUMO
Na determinação dos teores de carbono (C) em resíduos orgânicos, são utilizadas variações de marchas analíticas empregadas para solos, sem a necessária calibração. Assim, torna-se necessário avaliar e calibrar métodos de determinação dos teores de $\mathrm{C}$ e de matéria orgânica (MO) nesses materiais, tendo como referência o método da combustão seca. Objetivou-se neste estudo: avaliar métodos de determinação dos teores de carbono total (CT), carbono orgânico (CO) e MO em amostras de resíduos orgânicos diversificadas; determinar um modelo matemático de conversão de $\mathrm{C}$ e MO de um método analítico para outro; e avaliar a possibilidade de o método da mufla ser utilizado em rotina de laboratórios para análise de teores de MO e inferência de teores de CT em resíduos orgânicos. Foram avaliados os teores de CT, CO e MO em 42 amostras de compostos, estercos, resíduos vegetais, lodos de esgoto e camas de frango e de suíno, cujas coletas foram realizadas em 2010, em municípios do Sul de Minas Gerais e em Jundiaí-SP. As amostras foram secas em estufa a $65^{\circ} \mathrm{C}$, por $48 \mathrm{~h}$, maceradas e passadas em peneiras com malha de $0,250 \mathrm{~mm}$ e analisadas. Os métodos utilizados nas análises foram: Walkley-Black modificado - Yeomans \& Bremner (YB), combustão seca a $950^{\circ} \mathrm{C}$ (analisador TOC) e combustão em mufla a $550{ }^{\circ} \mathrm{C}$, para as determinações de CO, CT e MO, respectivamente. Os teores de CT nos resíduos orgânicos variaram de 8,5 a 51,4\%. $O$ método de Yeomans \& Bremner subestima os teores de $\mathrm{C}$ em relação ao método da combustão seca. Em função da determinação dos teores de MO e CO e de uso de equações matemáticas, é possível estimar os teores de CT pelo método da combustão seca.
\end{abstract}

Termos de indexação: analisador de carbono, combustão seca, Walkley-Black modificado.

\footnotetext{
(1) Parte da Dissertação de Mestrado do primeiro autor apresentada ao programa de Pós-Graduação em Ciência do Solo da Universidade Federal de Lavras - UFLA. Recebido para publicação em 27 de setembro de 2011 e aprovado em 18 de maio de 2012.

(2) Doutorando em Ciência do Solo, Universidade Federal de Lavras - UFLA. Caixa Postal 3037, CEP $37200-000$ Lavras (MG). Bolsista do CNPq. E-mail: davigoldan@yahoo.com.br

(3) Professor do Departamento de Ciência do Solo, UFLA. E-mail: csilva@dcs.ufla.br
} 


\title{
SUMMARY: QUANTIFICATION METHODS OF CARBON AND ORGANIC MATTER IN ORGANIC RESIDUES
}

\begin{abstract}
In the determination of carbon contents in organic residues, analytical methods for soils are frequently employed without adequate calibration. Thus, methods capable to determine the levels of $C$ and organic matter (OM) in organic residues must be tested and calibrated, using as a reference the dry combustion method. The objectives of this study were to evaluate methods for determining the levels of total carbon (TC), organic carbon (OC) and OM in organic residue samples; to determine mathematical equations for the conversion of $O M$ and $O C$ in TC quantified by the dry combustion method and to assess the viability to the muffle method for the routine of laboratories for OM quantification and to infere the TC contents in organic residues. In 2010, samples from 42 composts, crop residues, manures, sewage sludge, coffee husk, and sawdust, among others were collected in municipalities of the Southern region of Minas Gerais and in Jundiai-São Paulo. The samples were dried at $65^{\circ} \mathrm{C}$ for 48 hours, crushed and sieved $(0.250 \mathrm{~mm}$ opening) and analyzed. The following methods were tested: modified Walkley-Black, Yeomans and Bremner (YB), dry combustion (TOC analyzer) and muffle $(M)$ combustion at $550^{\circ} \mathrm{C}$, for the determination of $\mathrm{OC}, \mathrm{TC}$, and $\mathrm{OM}$, respectively. TC contents in organic residues varied from 8.5 to $51.4 \%$. The Yeomans and Bremner (YB) method underestimates the $C$ content when compared to the dry combustion method. Based on the $O M$ and $O C$ contents and mathematical equations, the TC combustion method contents can be estimated.
\end{abstract}

Index terms: carbon analyzer, dry combustion, modified Walkley-Black.

\section{INTRODUÇÃO}

O teor de carbono (C) é útil para avaliar o grau de humificação dos resíduos, uma vez que, com o aumento do tempo de compostagem, ocorre diminuição do teor de MO do composto (Dias et al., 2010). Também permite determinar a relação $\mathrm{C} / \mathrm{N}$, que é um dos fatores reguladores do processo de compostagem (Jiménez \& García, 1989). A determinação do C em resíduos orgânicos subsidia estudos que quantificam gases-estufa em pátios de compostagem, além de ser preditora do grau e da velocidade de decomposição dos resíduos compostados (Sánchez-Monedero et al., 2010). Em função das mudanças climáticas e do aquecimento global e do maior uso de resíduos na adubação das lavouras, o estudo de métodos precisos para determinar o C em resíduos orgânicos constitui-se em demanda recorrente de vários setores.

$\mathrm{A}$ análise do teor de $\mathrm{C}$ envolve a conversão em $\mathrm{CO}_{2}$ de todas as substâncias orgânicas presentes no resíduo, por meio de combustão seca ou úmida, por digestão, oxidação e dosagem do agente oxidante remanescente, por titulometria, volumetria, entre outros procedimentos analíticos (Gatto et al., 2009). Ao se avaliar o teor de $\mathrm{C}$, as variações nos resultados podem ocorrer tanto em razão dos métodos empregados, quanto em relação à composição química dos materiais. Além disso, para compostos, há diferenças nos fatores de conversão dos teores de C em MO (Jiménez \& García, 1992). Os métodos automatizados demandam menor quantidade de amostra, empregam reagentes analíticos certificados e de elevada pureza e, por isso, são utilizados como referência (Soon \& Abboud, 1991). No entanto, possuem custo elevado de análise e de manutenção dos equipamentos, daí a necessidade de utilizar rotinas analíticas que sejam de baixo custo na execução e que possam ser executadas na maioria dos laboratórios, sendo exemplos desses protocolos os métodos da mufla e de Walkley-Black modificado.

O método de Yeomans \& Bremner (1988) é uma variação do método de Walkley-Black e baseia-se na redução do dicromato $\left(\mathrm{Cr}_{2} \mathrm{O}_{7}^{2-}\right)$ por compostos de carbono orgânico e na subsequente determinação do $\mathrm{Cr}_{2} \mathrm{O}_{7}{ }^{2-}$ remanescente por titulação do excesso de cromo com sulfato ferroso amoniacal (Cantarella et al., 2001). Esse método é o mais empregado em laboratórios que analisam $\mathrm{C}$ em solos, porém apresenta problemas analíticos e ambientais, em razão da utilização do cromo (Segnini et al., 2008). O método da mufla consiste na determinação gravimétrica do $\mathrm{CO}_{2}$ evoluído e, por conseguinte, na perda de massa de resíduo submetido à alta temperatura por certo intervalo de tempo; na determinação da MO, considera-se, assim, a diferença de peso inicial (amostras secas a $105^{\circ} \mathrm{C}$ ) e de peso computado após a incineração da amostra a 550-600 ${ }^{\circ} \mathrm{C}$ (Suguio, 1973).

Para resíduos orgânicos, o emprego do método da mufla permite estimar o teor de $\mathrm{C}$ total de resíduos compostados, bastando para isso que se utilize o fator de 1,8 para conversão de MO em C total, conforme sugerem Jiménez \& García (1992). Para amostras de solo, Pribyl (2010) sugere um fator de 2, afirmando ser esse valor mais preciso que o fator "van Bemmelen" de 1,724, comumente usado para estimar o teor de MO em solos, a partir de C. O método da mufla também tem como vantagem ser simples e rápido; entretanto, os resultados obtidos são extremamente 
dependentes da temperatura final utilizada na execução do método, pois, quanto maior a temperatura, maior é a queima do material orgânico e menor o peso do material remanescente, o que implica variações nos fatores de conversão (Conceição et al., 1999). Assim, essas variações nos fatores de conversão sinalizam para a necessidade de realizar estudos que determinem o grau de correlação entre um método e outro e que possibilitem a conversão de $\mathrm{MO}$ em CT e CO, para resíduos orgânicos com diferentes origens e composição química.

O termo "resíduos orgânicos" abrange desde materiais compostados até materiais mais lábeis ou não decompostos, sendo exemplos os restos de culturas, resíduos vegetais, estercos, lodo de esgoto, compostos e os subprodutos industriais e agroindustriais. Esses materiais apresentam grande amplitude de teores de $\mathrm{C}$ e $\mathrm{N}$, os quais variam na faixa de 6,9 a $54,7 \%$ (Jiménez et al., 1992; Rodella \& Alcarde, 1994; Unsal \& Ok, 2001) e de 0,6 a 5,3 \% (Pascual et al., 1997; Unsal \& Ok, 2001; Melo et al., 2008), respectivamente. Essas variações podem ser justificadas pelo fato de serem resíduos provenientes de diversas origens e de processos industriais variados.

Diversos trabalhos evidenciam ser possível a determinação dos teores de $\mathrm{C}$ em resíduos orgânicos em razão de adaptações de métodos empregados na análise de solos (Rodella \& Alcarde, 1994; Castílhos et al., 2008; Melo et al., 2008). No entanto, na literatura relativa à determinação de $\mathrm{C}$ em resíduos orgânicos diversificados, não há métodos oficiais, tampouco fatores de conversão ou equações matemáticas que possibilitem converter $\mathrm{C}$ e $\mathrm{MO}$ de um método para outro.

Objetivou-se neste estudo: avaliar métodos de determinação dos teores de CT, CO e MO em amostras de resíduos orgânicos; disponibilizar equações matemáticas para a conversão de $\mathrm{C}(\mathrm{MO})$ entre os métodos; e avaliar a possibilidade de o método da mufla ser utilizado em rotina de laboratórios para análise de teores de MO e inferência dos teores de CT em resíduos orgânicos.

\section{MATERIAL E MÉTODOS}

As amostras de resíduos orgânicos são provenientes de municípios do Sul de Minas Gerais e de JundiaíSP. Foram utilizadas 42 amostras de resíduos (Quadro 1), visando cobrir ampla faixa de variação de teores de C nesses materiais. Após a etapa de coleta, as amostras foram armazenadas em sacos plásticos e, posteriormente, maceradas, passadas em peneiras com malha de $0,250 \mathrm{~mm}$ de abertura e secas em estufa de circulação forçada de ar a $65{ }^{\circ} \mathrm{C}$, por $48 \mathrm{~h}$. A seguir, foram identificadas e armazenadas em dessecador, até a execução dos protocolos de determinação dos teores de $\mathrm{MO}$ e de $\mathrm{C}$ nos resíduos. As marchas analíticas utilizadas para determinação dos teores de $\mathrm{MO}$ e de $\mathrm{C}$ nos resíduos são as descritas a seguir.

\section{Método de Yeomans \& Bremner (YB)}

Para execução da marcha analítica de Yeomans \& Bremner (1988), foram realizadas as seguintes adaptações: em tubo de digestão, foi pesado $0,1 \mathrm{~g}$ de cada resíduo orgânico. Em seguida, foram adicionados $5 \mathrm{~mL}$ de $\mathrm{K}_{2} \mathrm{Cr}_{2} \mathrm{O}_{7} 0,167 \mathrm{~mol} \mathrm{~L}^{-1}$ e $10 \mathrm{~mL}$ de $\mathrm{H}_{2} \mathrm{SO}_{4}$ concentrado. Posteriormente, as amostras em tubos foram acondicionadas em bloco digestor e digeridas em temperatura de $170{ }^{\circ} \mathrm{C}$, por $30 \mathrm{~min}$; após essa etapa e depois de serem resfriadas, as amostras digeridas foram transferidas para erlenmeyers, onde foram adicionados $5 \mathrm{~mL}$ de $\mathrm{H}_{3} \mathrm{PO}_{4}$, visando facilitar a visualização do ponto de viragem da titulação. Em seguida, foram adicionadas três gotas do indicador difenilamina $1 \%$, sendo as amostras tituladas com sulfato ferroso amoniacal $0,4 \mathrm{~mol} \mathrm{~L}^{-1}$. Os teores de carbono orgânico (CO) foram determinados utilizandose os fundamentos e equação matemática descritos em Cantarella et al. (2001).

\section{Método da mufla}

A determinação do teor de matéria orgânica pelo método da mufla foi feita seguindo-se método estabelecido por Goldin (1987), com as seguintes modificações: secagem prévia das amostras em estufa a $105{ }^{\circ} \mathrm{C}$, por um período de $24 \mathrm{~h}$, visando eliminar toda a água presente nos resíduos, como a higroscópica, a capilar ou de cristalização (Rodella \& Alcarde, 1994). Após esse período, os cadinhos de cerâmica com as amostras foram acondicionados em forno do tipo mufla e incinerados em uma temperatura de $550^{\circ} \mathrm{C}$, por $3 \mathrm{~h}$. Posteriormente, o conjunto (cadinho+resíduos) foi acondicionado em dessecador e, em seguida, pesado.

$\mathrm{O}$ teor de matéria orgânica foi determinado em razão da perda de massa do resíduo incinerado, considerando-se o material perdido pela queima no intervalo de variação da temperatura de $105^{\circ} \mathrm{C}$ a $550^{\circ} \mathrm{C}$, conforme a fórmula: $\mathrm{MO}(\%)=(\mathrm{P}-(\mathrm{T}-\mathrm{C}) \mathrm{x}$ $100) / \mathrm{P}$, em que $\mathrm{P}=$ peso da amostra $(\mathrm{g})$ depois de aquecida a $105^{\circ} \mathrm{C} ; \mathrm{C}=$ tara do cadinho $(\mathrm{g}) ; \mathrm{e} \mathrm{T}=$ peso da cinza + cadinho $(\mathrm{g})$.

\section{Método da combustão seca}

$\mathrm{A}$ análise do $\mathrm{C}$ total dos resíduos foi feita por combustão seca em analisador marca Elementar, modelo Vario TOC, sendo as pesagens das amostras (2 a $5 \mathrm{mg}$ ) de cada resíduo realizadas em balança analítica da marca METTLER TOLEDO, do nível "Classic", com precisão de pesagem de 0,00001 g. Os resíduos orgânicos utilizados foram macerados em almofariz e peneirados (malha de 0,250 mm); em seguida, foram secos em estufa a $65^{\circ} \mathrm{C}$, durante $48 \mathrm{~h}$, para retirar a umidade, antes da pesagem e análise no analisador TOC. As amostras foram acondicionadas e seladas em cápsulas de estanho, sendo, em seguida, incineradas em temperatura aproximada de $950{ }^{\circ} \mathrm{C}$, 
Quadro 1. Amostras de resíduos orgânicos utilizadas nas marchas de quantificação dos teores de C e de MO

\begin{tabular}{|c|c|c|c|}
\hline Amostra & Resíduo & Amostra & Resíduo \\
\hline 1 & Cama de frango (a) & 22 & Composto (d) \\
\hline 2 & Cama de frango (b) & 23 & Composto (e) \\
\hline 3 & Composto (a) & 24 & Esterco de galinha (a) \\
\hline 4 & Esterco: Galinha+Codorna & 25 & Substrato comercial \\
\hline 5 & Esterco de cavalo & 26 & Composto (f) \\
\hline 6 & Esterco seco (a) & 27 & Composto (g) \\
\hline 7 & Esterco seco (b) & 28 & Lodo de esgoto (a) \\
\hline 8 & Casca de pinus (a) & 29 & Cama de frango (c) \\
\hline 9 & Cama de suíno (a) & 30 & Lodo de esgoto (b) \\
\hline 10 & Casca de café+Esterco Suíno & 31 & Pergaminho de café \\
\hline 11 & Casca de coco & 32 & Casca de café (lavador) \\
\hline 12 & Composto (b) & 33 & Esterco de codorna (b) \\
\hline 13 & Esterco de suíno & 34 & Casca de pinus (b) \\
\hline 14 & Esterco de codorna (a) & 35 & Cama de frango (d) \\
\hline 15 & Esterco úmido & 36 & Casca de café em coco \\
\hline 16 & Serragem & 37 & Esterco de galinha (b) \\
\hline 17 & Composto (c) & 38 & Fibra de coco \\
\hline 18 & Esterco de aves & 39 & Turfa \\
\hline 19 & Cama de suíno (b) & 40 & Lodo de esgoto (c) \\
\hline 20 & Lodo de biodigestor & 41 & Casca de café \\
\hline 21 & Esterco bovino & 42 & Lodo de esgoto compostado \\
\hline
\end{tabular}

por 5 min, em um tubo de quartzo de combustão, sendo utilizado como carreador o gás $\mathrm{O}_{2}$ com $99,998 \%$ de pureza. Após a combustão, toda a matéria orgânica foi convertida em $\mathrm{CO}_{2}$ e um sensor infravermelho detectou a quantidade de dióxido de carbono $\left(\mathrm{CO}_{2}\right)$ gerado pela combustão, relacionando-a automaticamente com a quantidade de $\mathrm{C}$ elementar existente na amostra. $\mathrm{Na}$ confecção de curva-padrão de $\mathrm{C}$ para o analisador automático, foram utilizadas amostras puras para análise de PHP (Potassium hydrogen phthalate, 47,05 $\%$ de $\mathrm{C})$ e de sucrose $(42,1 \%$ de $\mathrm{C})$.

\section{Determinação de C-substâncias húmicas, índice de humificação e carbono residual}

Para quantificação dos teores de C-substâncias húmicas nos resíduos, foi utilizado o método de extração e fracionamento quantitativo descrito em Benites et al. (2003), que se baseia na solubilidade diferenciada das substâncias húmicas em meios alcalino e ácido. Após o fracionamento, determinaramse os teores de $\mathrm{C}$ nas frações húmicas (ácido húmico e ácido fúlvico) e o C-residual = CO - (C-fração ácido húmico + C-fração ácido fúlvico), de acordo com Yeomans \& Bremner (1988). O índice de humificação (IH) foi calculado de acordo com procedimento sugerido por Jouraiphy et al. (2005), que preconizam o cálculo da seguinte razão: C-fração ácido húmico (C-FAH)/ $\mathrm{CO}$, em percentagem.

\section{Análise estatística}

Os resultados foram submetidos à análise de regressão, e os parâmetros das equações matemáticas foram testados quanto ao grau de significância pelo teste $t(p<0,01)$.

\section{RESULTADOS E DISCUSSÃO}

Os teores de MO, CT e CO para diferentes resíduos orgânicos, determinados pelos métodos da mufla, da combustão seca e de YB, respectivamente, são apresentados no quadro 2 . Os teores de $\mathrm{MO}, \mathrm{CT}$ e CO variaram de 18,8 a $99,4 \%, 8,5$ a $51,4 \%$ e 6,1 a 45,6 $\%$, respectivamente, refletindo a heterogeneidade das amostras analisadas e as diferenças entre os métodos analíticos. Considerando-se como referência o método da combustão seca, pela sua exatidão, as amostras de resíduos orgânicos analisadas apresentaram ampla faixa de teores de CT - fato relevante para o estudo de métodos de determinação de $\mathrm{C}$.

Observou-se que o método YB propiciou recuperação média de $90 \%$ dos teores de CT determinados pelo método da combustão seca, considerando-se o fator de conversão (Quadro 2). Esse resultado está em acordo com os dados de Gatto et al. 
Quadro 2. Teores de matéria orgânica (MO), carbono total (CT) e carbono orgânico (CO) determinados, respectivamente, pelos métodos mufla, combustão seca e Yeomans \& Bremner (YB), para amostras diversificadas de resíduos orgânicos

\begin{tabular}{|c|c|c|c|c|c|c|}
\hline \multirow[t]{2}{*}{ Amostra } & \multicolumn{3}{|c|}{ Teor $(\%)$} & \multicolumn{3}{|c|}{ Fator } \\
\hline & MO & CT & CO & MO/CT & MO/CO & $\mathrm{CO} / \mathrm{CT}$ \\
\hline 1 & 84,3 & 36,7 & 31,9 & 2,3 & 2,6 & 0,87 \\
\hline 2 & 78,8 & 31,5 & 27,9 & 2,5 & 2,8 & 0,89 \\
\hline 3 & 67,6 & 31,9 & 28,1 & 2,1 & 2,4 & 0,88 \\
\hline 4 & 54,9 & 27,3 & 24,5 & 2,0 & 2,2 & 0,90 \\
\hline 5 & 87,7 & 41,4 & 38,7 & 2,1 & 2,3 & 0,94 \\
\hline 6 & 79,1 & 32,9 & 30,5 & 2,4 & 2,6 & 0,93 \\
\hline 7 & 78,8 & 35,6 & 32,9 & 2,2 & 2,4 & 0,93 \\
\hline 8 & 98,1 & 46,6 & 39,2 & 2,1 & 2,5 & 0,84 \\
\hline 9 & 69,9 & 23,9 & 17,0 & 2,9 & 4,1 & 0,71 \\
\hline 10 & 91,6 & 44,6 & 35,9 & 2,1 & 2,6 & 0,80 \\
\hline 11 & 49,4 & 18,2 & 16,1 & 2,7 & 3,1 & 0,88 \\
\hline 12 & 58,5 & 25,8 & 23,2 & 2,3 & 2,5 & 0,90 \\
\hline 13 & 68,3 & 30,5 & 25,7 & 2,2 & 2,7 & 0,84 \\
\hline 14 & 69,7 & 28,8 & 25,7 & 2,4 & 2,7 & 0,89 \\
\hline 15 & 62,4 & 27,7 & 24,5 & 2,3 & 2,5 & 0,89 \\
\hline 16 & 99,4 & 48,2 & 45,6 & 2,1 & 2,2 & 0,95 \\
\hline 17 & 44,1 & 20,0 & 19,4 & 2,2 & 2,3 & 0,97 \\
\hline 18 & 61,7 & 28,6 & 22,2 & 2,2 & 2,8 & 0,78 \\
\hline 19 & 91,1 & 39,0 & 33,3 & 2,3 & 2,7 & 0,85 \\
\hline 20 & 66,6 & 32,2 & 30,7 & 2,1 & 2,2 & 0,95 \\
\hline 21 & 63,7 & 27,9 & 25,6 & 2,3 & 2,5 & 0,92 \\
\hline 22 & 36,4 & 18,0 & 13,1 & 2,0 & 2,8 & 0,73 \\
\hline 23 & 33,8 & 16,6 & 12,6 & 2,0 & 2,7 & 0,76 \\
\hline 24 & 38,6 & 21,2 & 11,7 & 1,8 & 3,3 & 0,55 \\
\hline 25 & 48,0 & 19,7 & 17,5 & 2,4 & 2,7 & 0,89 \\
\hline 26 & 39,8 & 19,9 & 16,2 & 2,0 & 2,5 & 0,82 \\
\hline 27 & 67,7 & 27,1 & 27,1 & 2,5 & 2,5 & 1,00 \\
\hline 28 & 20,9 & 12,9 & 8,5 & 1,6 & 2,5 & 0,66 \\
\hline 29 & 81,2 & 31,8 & 31,2 & 2,6 & 2,6 & 0,98 \\
\hline 30 & 54,1 & 24,7 & 23,5 & 2,2 & 2,3 & 0,95 \\
\hline 31 & 97,2 & 45,7 & 41,4 & 2,1 & 2,3 & 0,91 \\
\hline 32 & 88,7 & 41,5 & 37,9 & 2,1 & 2,3 & 0,91 \\
\hline 33 & 72,9 & 33,7 & 29,6 & 2,2 & 2,5 & 0,88 \\
\hline 34 & 98,8 & 51,4 & 42,1 & 1,9 & 2,3 & 0,82 \\
\hline 35 & 75,2 & 32,9 & 27,5 & 2,3 & 2,7 & 0,84 \\
\hline 36 & 92,2 & 44,1 & 37,5 & 2,1 & 2,5 & 0,85 \\
\hline 37 & 64,2 & 27,3 & 20,6 & 2,4 & 3,1 & 0,76 \\
\hline 38 & 98,0 & 48,3 & 39,5 & 2,0 & 2,5 & 0,82 \\
\hline 39 & 18,8 & 8,5 & 6,1 & 2,2 & 3,1 & 0,72 \\
\hline 40 & 49,2 & 20,7 & 19,4 & 2,4 & 2,5 & 0,94 \\
\hline 41 & 91,3 & 43,1 & 36,2 & 2,1 & 2,5 & 0,84 \\
\hline \multirow[t]{2}{*}{42} & 54,7 & 27,4 & 25,4 & 2,0 & 2,2 & 0,93 \\
\hline & & & $\mathrm{MD}$ & $2,2 \pm 0,2$ & $2,6 \pm 0,3$ & $0,9 \pm 0,1$ \\
\hline
\end{tabular}

MD = média dos fatores e desvio-padrão, respectivamente. 
(2009), que, empregando o método de YB, verificaram que os teores de $\mathrm{C}$ em solo foram subestimados em relação ao método da combustão seca, o que, possivelmente, se deve ao fato de, ao se utilizar a oxidação com dicromato de potássio, ocorrer apenas a oxidação parcial do $\mathrm{C}$, tendo em vista que $\mathrm{C}$-frações orgânicas protegidas pela fase mineral, C-carbonato e C-carvão não são atacados durante a digestão ácida (Cantarella \& Trivelin, 2001; Segnini et al., 2008). Resultados semelhantes foram obtidos por Rheinheimer et al. (2008) e Segnini et al. (2008), analisando solos e utilizando métodos que envolvem a oxidação do $\mathrm{CO}$ pelo dicromato em meio ácido. Além do que foi dito, os teores de $\mathrm{CO}$ determinados pelo método YB podem sofrer interferências de vários constituintes da amostra, como de íons ferrosos, sulfeto, N-nítrico, cloreto e óxido de manganês, os quais podem ser oxidados ou reduzidos. Além disso, não se pode assumir que todo $\mathrm{CO}$ da amostra possua valência zero (Rodella \& Alcarde, 1994; Nelson \& Sommers, 1996). É fato que alguns resíduos orgânicos possuem C inorgânico, principalmente os estercos de aves, visto que o uso de corretivos de acidez é frequente em criatórios. Nesse sentido, o pré-tratamento das amostras (etapa não realizada neste estudo) com ácido contribui para a retirada do $\mathrm{C}$ inorgânico (Kerven et al., 2000). Ademais, a precisão na determinação dos teores de $\mathrm{CO}$ pode ser alterada também por erros de diluições durante a realização das análises ou de visualização de ponto de viragem durante a titulação, de modo que esses fatores podem causar erros na determinação dos teores de C (Conceição et al., 1999).

Em razão de o método de $\mathrm{YB}$ ser baseado na oxidação do C pelo dicromato, Nelson \& Sommers (1996) relatam que as frações orgânicas mais resistentes à degradação, como a humina, não são oxidadas pelo dicromato. Baseando-se nessa premissa e utilizandose os dados de amostras de resíduos humificadas (amostras 3, 12, 17, 20, 28, 30, 39, 40 e 42), não foi observada relação do teor de C-substâncias húmicas, índice de humificação e C-residual com a recuperação do $\mathrm{C}$ pelo método YB(Figuras 1a, b, c). Esse resultado, possivelmente, indica que a resistência à oxidação deve-se a outros fatores, como a proteção da matéria orgânica por minerais (Eusterhues et al., 2003) e a recalcitrância química específica de frações orgânicos dos resíduos (Eusterhues et al., 2005). O fato de o CSHs abranger, neste estudo, somente o C-fração ácido húmico e C-fração ácido fúlvico podem, também, explicar as baixas relações do C-residual com o C recuperado em função do emprego do dicromato de potássio. Outro ponto a ser considerado é a presença de frações de C carbonizadas nos resíduos, o que aumenta o grau de recalcitrância da matéria orgânica. Além disso, o universo de amostras humificadas aqui analisadas foi reduzido (nove amostras, de um total de 42), sendo necessário, em estudos futuros, ampliar o número de amostras humificadas e o escopo de análises de frações mais recalcitrantes, a fim de que seja elucidada a relação entre a recuperação de $\mathrm{C}$ pelas
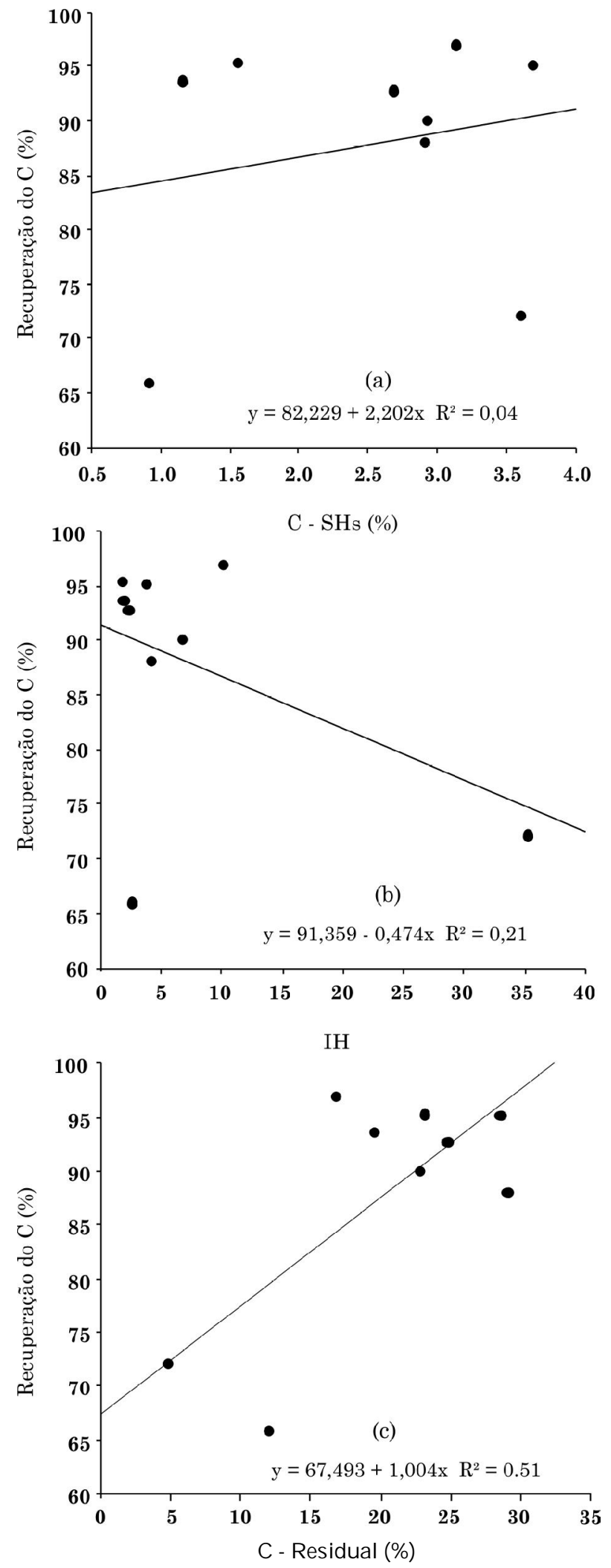

Figura 1. Relações entre teor de C-substâncias húmicas (C-SHs = C-fração ácido húmico + Cfração ácido fúlvico), índice de humificação (IH) e C-residual com a recuperação do carbono $(\mathrm{CO} /$ CT x 100), em resíduos orgânicos humificados. 
variantes do método de Walkley-Black e os pools recalcitrantes de $\mathrm{C}$ presentes nos resíduos orgânicos.

Observa-se que, em função da análise dos dados mostrados no quadro 2 , os fatores médios $(2,2$ e 0,9$)$ obtidos pelas relações $\mathrm{MO} / \mathrm{CT}$ e $\mathrm{CO} / \mathrm{CT}$, respectivamente, apresentaram resultados mais satisfatórios, com menores valores de desvio-padrão, quando comparados ao fator $2,6(\mathrm{MO} / \mathrm{CO})$, mostrando que há maior variabilidade e imprecisão da relação $\mathrm{MO} /$ CO. Esse resultado deveu-se, provavelmente, ao fato de a oxidação do CT ser mais eficiente quando se adota o procedimento de combustão seca, quando comparado com a determinação de $\mathrm{CO}$ pelo método de YB. Outro fator que se deve levar em consideração é que o método da combustão seca, em razão da queima das amostras a $950{ }^{\circ} \mathrm{C}$, propicia tanto a evolução do $\mathrm{C}$ inorgânico quanto do orgânico a $\mathrm{CO}_{2}$, ao passo que o método $\mathrm{YB}$ recupera somente o C orgânico ou oxidável (Nelson \& Sommers, 1996), que possui graus variados de recuperação e depende da amostra analisada.

As razões $\mathrm{MO} / \mathrm{CT}, \mathrm{MO} / \mathrm{CO}$ e $\mathrm{CO} / \mathrm{CT}$ apresentam variações em seus valores de um resíduo para outro, porém as médias e desvios-padrão obtidos estão dentro de faixa de valores considerados aceitáveis para esse tipo de estudo. Pascual et al. (1997) encontraram fator médio de 2,05 para a razão $\mathrm{MO} / \mathrm{CO}$, em vez de 1,724 , que é normalmente utilizado para estimar o teor de MO em solo, a partir do teor de CO, não sendo, assim, adequado o uso desse fator para resíduos orgânicos. Ao comparar diferentes métodos de determinação do $\mathrm{C}$, em solos com altos teores de MO, Pereira et al. (2006) sugeriram um fator de 2,1 para a conversão de MO em CO, utilizando o método Walkley \& Black (1934), modificado por Tedesco et al. (1995), que tem o mesmo princípio do método de Yeomans \& Bremner (1988). Em revisão ampla sobre o tema, Pribyl (2010) sugere a adoção de fator igual a 2 , baseando-se na premissa de que a $\mathrm{MO}$ apresenta $50 \%$ de $\mathrm{C}$, sendo esse fator, em quase todos os estudos analisados, mais acurado que o fator de 1,724.

No estudo de Melo et al. (2008), em acordo com os resultados de Pascual et al. (1997), foi observado fator médio de 2,06 para a relação MO/CO, com variação de 1,80 a 2,36 , para diversas amostras de resíduos orgânicos, com exceção do lodo de esgoto 2 , o que difere dos fatores encontrados neste trabalho. Os autores relatam ainda que, no caso de resíduos de diferentes origens, torna-se difícil estabelecer um fator confiável para estimar a $\mathrm{MO}$ a partir do CO, e vice-versa, o que sinaliza para o uso de equações matemáticas nesse tipo de abordagem, as quais proporcionam maior precisão nas interconversões de $\mathrm{MO}$ a C, ou vice-versa, como se propõe no presente estudo. Esse tipo de abordagem está em acordo com Navarro et al. (1993), que sugerem as seguintes equações para o mesmo fim: $\mathrm{CT}=0,51$ (MO -1) +0,48 e CO =0,51 (MO - 1) - 3,59, para determinação do CT e CO, respectivamente, a partir do teor de MO.

As equações matemáticas que relacionam CT, CO e MO são apresentadas na figura 2. Na figura 2a é apresentada a equação de regressão entre $\mathrm{CO}$ e $\mathrm{MO}$, a qual mostrou alto coeficiente de determinação $\left(R^{2}=\right.$ $0,92)$ e elevada significância ( $p<0,01)$. Em razão desses resultados, pode-se inferir que o teor de $\mathrm{CO}$ pode ser estimado em função do teor de MO determinado pelo método da mufla, mediante o uso da seguinte equação: $\mathrm{CO}=0,425 \mathrm{MO}$ - 2,064. A regressão entre CT e MO é apresentada na figura $2 \mathrm{~b}$, de modo que esse modelo matemático pode ser usado para inferir o teor de CT em função do teor de MO determinado pelo método da mufla, devido ao alto coeficiente de determinação obtido $\left(R^{2}=0,93\right)$ e à elevada significância $(p<0,01)$, mediante utilização da seguinte equação: $\mathrm{CT}=0,463 \mathrm{MO}$ - 0,550. O método da mufla é vantajoso em relação aos outros (combustão seca e YB), pois, além de não gerar resíduos contaminados, é rápido, de fácil operação e de baixo custo (Pribyl, 2010), aspectos relevantes na determinação da MO em análises de rotina. Tendo em vista os resultados apresentados, é possível utilizar o teor de MO-mufla para calcular o teor de CT pelo método da combustão seca.

Apesar das vantagens citadas, o teor de MO determinado pelo método da mufla pode ser superestimado, por se basear na diferença de peso, ou seja, a massa perdida em temperatura de incineração $\left(110\right.$ a $\left.550{ }^{\circ} \mathrm{C}\right)$ é considerada como $\mathrm{MO}$, de modo que a degradação de frações minerais pode resultar em erro na determinação do CT e CO, pois as massas dessas substâncias inorgânicas são computadas como matéria orgânica (Rodella \& Alcarde, 1994; Leong \& Tanner, 1999). Assim, para que haja precisão nos resultados de MO obtidos pela mufla, torna-se necessário que os resíduos orgânicos não sejam contaminados com solo e outros condicionadores químicos (gesso, cal hidratada, calcário, superfosfato simples, entre outros), os quais, muitas vezes, são adicionados a camas e estercos de animais, visando melhorar a qualidade física, química e biológica desses subprodutos, de modo que esses "contaminantes" podem influenciar e causar erros nos resultados analíticos obtidos.

$\mathrm{Na}$ figura 2c é apresentada a relação entre os teores de $\mathrm{CO}$ e CT obtidos pelos métodos de YB e de combustão seca, respectivamente. Em razão do alto coeficiente de determinação $\left(\mathrm{R}^{2}=0,95\right)$, a $1 \%$ de probabilidade, os teores de $\mathrm{C}$ de um método para outro podem ser convertidos, utilizando a seguinte equação: $\mathrm{CO}=0,900 \mathrm{CT}-1,010$. Observa-se que o coeficiente angular da regressão linear $(0,900)$ é inferior a 1 , indicando que o método $\mathrm{YB}$ subestimou os teores de $\mathrm{C}$, em relação ao $\mathrm{C}$ determinado pela combustão seca. Já foi mencionado que o método YB não se presta à determinação do $\mathrm{C}$ inorgânico (Tabatabai, 1996), que pode estar presente nos resíduos na forma de carbonatos, óxidos e hidróxidos de Ca e Mg; por sua vez, o método de combustão presta-se à determinação do $\mathrm{CT}$, que é constituído de C inorgânico + orgânico; na análise por combustão seca, o carbonato evolui a $\mathrm{CO}_{2}$ e, em seguida, é quantificado por espectroscopia de absorção no infravermelho e transformado em $\mathrm{C}$ total; além do $\mathrm{C}$ 

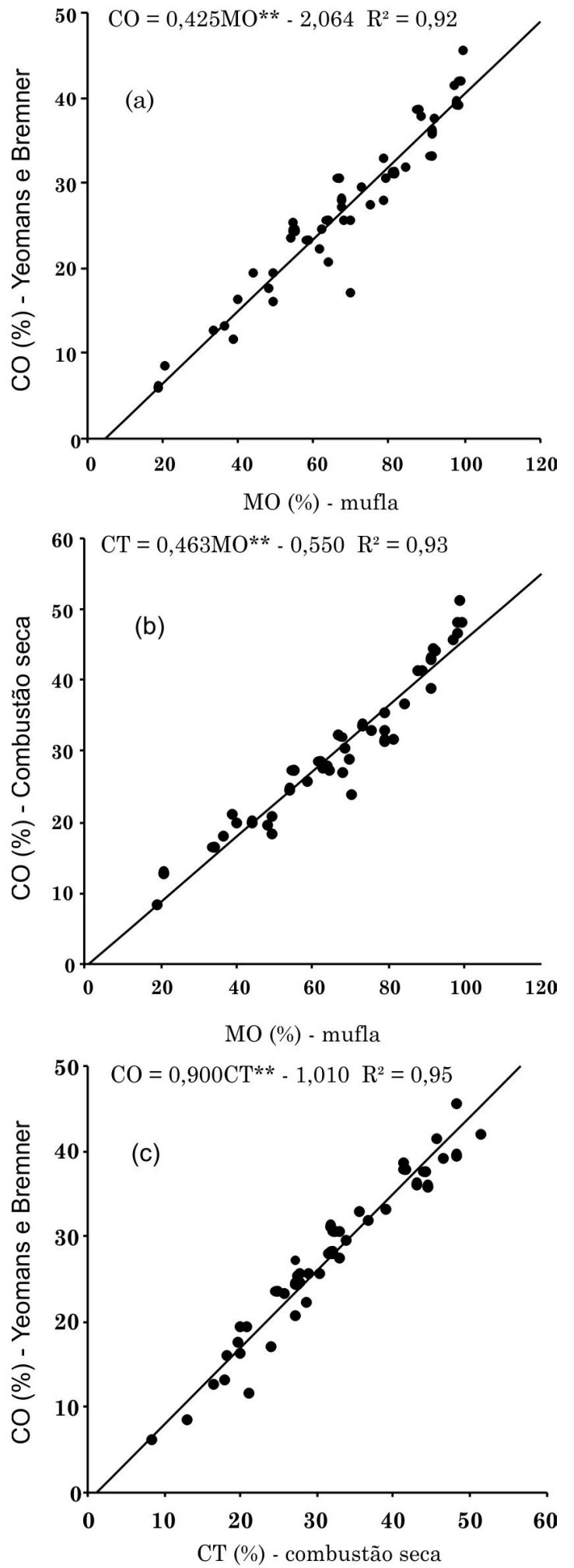

Figura 2. Relação entre os teores de (a) CO e de MO, (b) CT e MO e (c) CO e CT, obtidos pelos métodos de Yeomans \& Bremner, da mufla e da combustão seca, para os teores de CO, MO e CT, respectivamente, para as 42 amostras de resíduos orgânicos analisadas. $\mathrm{CO}=$ carbono orgânico, $\mathbf{C T}=$ carbono total e MO = matéria orgânica. **: significativo a p $<0,01$. inorgânico, em razão da temperatura de combustão, mesmo as formas mais recalcitrantes e carbonizadas são convertidas em $\mathrm{CO}_{2}$, de modo que a recuperação de $\mathrm{C}$ é plena pelo método da combustão seca.

Os dados obtidos neste estudo mostram que o método de Yeomans \& Bremner subestima os teores de $\mathrm{C}$, tendo o método da combustão seca como referência. É possível utilizar o método da mufla para avaliar o teor de $\mathrm{MO}$ e, a partir desse resultado, para estimar o teor de CT em resíduos diversos. Foram obtidas regressões lineares com altos coeficientes de determinação, o que permite estimar os teores de CT a partir da determinação dos teores de CO e MO. Apesar do elevado grau de associação entre MO e CO, não é razoável utilizar a $\mathrm{MO}$ para estimar o $\mathrm{CO}$, ou viceversa, em razão dos erros e do fato de nem o método da mufla nem o de Yeomans \& Bremner serem considerados de referência, como é o caso do método da combustão seca.

\section{CONCLUSÕES}

1. Os teores de carbono total nos resíduos orgânicos apresentaram grande amplitude de variação, compreendendo valores na faixa de 8,5 a 51,4\%.

2. O método de Yeomans \& Bremner subestima os teores de carbono em relação ao método da combustão seca.

3. Equações de regressão linear com altos coeficientes de determinação permitem transformar com exatidão matéria orgânica em carbono total (CT = 0,463 MO - 0,550) e carbono orgânico em carbono total $(\mathrm{CO}=0,900 \mathrm{CT}-1,010)$.

4. Em razão do elevado grau de associação entre matéria orgânica e carbono total, é possível utilizar em rotina o método da mufla para avaliar o carbono presente em resíduos orgânicos de origens diversas.

\section{AGRADECIMENTOS}

À FAPEMIG (processo CAG - PPM-00424-09), pelo financiamento de ações de pesquisa deste estudo. Ao Dr. Fernando Carvalho Oliveira e Dr. Jonas Jacob Chiaradia (Biossolo Agricultura e Ambiente Ltda.) e à Opersan Serviços Ambientais, por fornecerem amostra de lodo de esgoto. Ao CNPq, pelo fornecimento de bolsas aos autores.

\section{LITERATURA CITADA}

BENITES, V.M.; MÁDARI, B. \& MACHADO, P.L.O.A. Extração e fracionamento quantitativo de substâncias húmicas do solo: um procedimento simplificado e de baixo custo. Rio de Janeiro, Embrapa, 2003. 7p. (Comunicado Técnico, 16) 
CANTARELlA, H.; QUAGGIO, J.A.; RAIJ, B. van. Determinação da matéria orgânica. In: RAIJ, B. van; ANDRADE, J.C.; CANTARELLA, H. \& QUAGGIO, J.A. Análise química pra avaliação da fertilidade de solos tropicais. Campinas, IAC, 2001. p.173-180.

CASTILHOS, R.M.V.; DICK, D.P.; CASTILHOS, D.D.; MORSELLI, T.B.A.G.; COSTA, P.F.P.; CASAGRANDE, W.B.C. \& ROSA, C.M. Distribuição e caracterização de substâncias húmicas em vermicompostos de origem animal e vegetal. R. Bras. Ci. Solo, 32:2669-2675, 2008.

CONCEIÇÃO, M.; MANZATTO, C.V.; ARAÚJO, W.S.; MARTIN NETO, L.; SAAB, S.C.; CUNHA, T.J.F. \& FREIXO, A.A. Estudo comparativo de métodos de determinação do teor de matéria orgânica em organossolos. Rio de Janeiro, Embrapa, 1999. 4p. (Comunicado Técnico, 2)

DIAS, B.O.; SILVA, C.A.; HIGASHIKAWA, F.S.; ROIG, A. \& SÁNCHEZ-MONEDERO, M.A. Use of biochar as bulking agent for the composting of poultry manure: Effect on organic matter degradation and humification. Biores. Technol., 101:1239-1246, 2010.

EUSTERHUES, K.; RUMPEL, C.; KLEBER, M. \& KOGELKNABNER, I. Stabilization of soil organic matter by interactions with minerals as revealed by mineral dissolution and oxidative degradation. Organ. Geochem., 34:1591-1600, 2003.

EUSTERHUES, K.; RUMPEL, C. \& KOGEL-KNABNER, I. Stabilization of soil organic matter isolated via oxidative degradation. Organ. Geochem., 36:1567$1575,2005$.

GATTO, A.; BARROS, N.F.; NOVAIS, R.F.; SILVA, I.R.; MENDONÇA, E.S. \& VILLANI, E.M.A. Comparação de métodos de determinação do carbono orgânico em solos cultivados com eucalipto. R. Bras. Ci. Solo, 33:735-740, 2009.

GOLDIN, A. Reassessing the use of loss-on-ignition for estimating organic matter content in noncalcareous soils. Commun. Soil Sci. Plant. Anal., 18:1111-1116, 1987.

JIMÉNEZ, E.I. \& GARCÍA, P. Evaluation of city refuse compost maturity: A review. Biol. Wastes, 27:115-142, 1989.

JIMÉNEZ, E.I. \& GARCIA, V.P. Relationships between organic carbon and total organic matter in municipal solid wastes and city refuse composts. Biores. Technol., 41:265$272,1992$.

JOURAIPHY, A.; AMIR, S.; EL GHAROUS, M.; REVEL, J. \& HAFIDI, M. Chemical and spectroscopic analysis of organic matter transformation during composting of sewage sludge and green plant waste. Inter. Biodet. Biodegr., 56:101-108, 2005.

KERVEN, G.L.; MENZIES, N.W. \& GEYER, M.D. Soil carbon determination by high temperature combustion - a comparison with dichromate oxidation procedures and the influence of charcoal and carbonate carbon on the measure value. Commun. Soil Sci. Plant Anal., 31:1935$1939,2010$.
LEONG, L.S. \& TANNER, P.A. Comparison of methods for determination of organic carbon in marine sediment. Marine Pollut. Bull., 38:875-879, 1999.

MELO, L.C.A.; SILVA, C.A. \& DIAS, B.O. Caracterização da matriz orgânica de resíduos de origens diversificadas. $R$. Bras. Ci. Solo, 32:101-110, 2008.

NAVARRO, A.F.; CEGARRA, J.; ROIG, A. \& GARCIA, D. Relationships between organic matter and carbon contents of organic wastes. Biores. Technol., 44:203-207, 1993.

NELSON, D.W.; SOMMERS, L.E. Total carbon, organic carbon, and organic matter. In: BLACK, C.A., ed. Methods of soil analysis. Chemical methods. Madison, Soil Science of America/American Society of Agronomy, 1996. Part 3. p.961-1010.

PASCUAL, J.A.; AYUSO, M.; GARCIA, C. \& HERNÁNDEZ, T. Characterization of urban wastes according to fertility and phytotoxicity parameters. Waste Manag. Res., 15:103$112,1997$.

PEREIRA, M.G.; VALLADARES, G.S.; ANJOS, L.H.C.; BENITES, V.M.; ESPÍNDULA JR., A. \& EBELING, A.G. Organic carbon determination in Histosols and soil horizons with high organic matter content from Brazil. Sci. Agric., 63:187-193, 2006.

PRIBYL, D.W. A critical review of the conventional SOC to SOM conversion factor. Geoderma, 156:75-83, 2010.

RHEINHEIMER, D.S.; CAMPOS, B.C.; GIACOMINI, S.J.; CONCEIÇÃO, P.C. \& BORTOLUZZI, E.C. Comparação de métodos de determinação de carbono orgânico total no solo. R. Bras. Ci. Solo, 32:435-440, 2008.

RODELLA, A.A. \& ALCARDE, J.C. Avaliação de materiais orgânicos empregados como fertilizantes. Sci. Agric., 51:556-562, 1994.

SÁNCHEZ-MONEDERO, M.A.; SERRAMIÁ, N.; CIVANTOS, C.G.O.; FERNÁNDEZ-HERNÁNDEZ, A. \& ROIG, A. Greenhouse gas emissions during composting of two-phase olive Mill wastes with different agroindustrial by-products. Chemosphere, $81: 18-25,2010$

SEGNINI, A.; SANTOS, L.M.; SILVA, W.T.L.; MARTINNETO, L.; BORATO, C.E.; MELO, W.J. \& BOLONHEZI, D. Estudo comparativo de métodos para a determinação da concentração de carbono em solos com altos teores de Fe (Latossolos). Química Nova., 31:94-97, 2008.

SOON, Y.K. \& ABBOUD, S. A comparison of some methods for soil organic carbon determination. Commun. Soil Sci. Plant. Anal., 22:943-954, 1991.

SUGUIO, K. Introdução à sedimentologia. São Paulo, Edgard Blücher, 1973. 317p.

TABATABAI, M.A. Soil organic matter testing: an overview. In: MAGDOFF, F.R.; TABATABAI, M.A. \& HANLON JR., E.A., eds. Soil organic matter: analysis and interpretation. Madison, Soil Science Society of America, 1996. p.1-9. (Special Publication, 46) 
TEDESCO, M.J.; GIANELlO, C.; BISSANI, C.A.; VOLKWEISS, S.J. Análises de solo, plantas e outros materiais. 2.ed. Porto Alegre: UFRGS, 1995. 174p.

UNSAL, T. \& OK, S.S. Description of characteristics of humic substances from different waste materials. Biores. Technol., 78:239-242, 2001.
WALKLEY, A. \& BLACK, I.A. An examination of the Degtjareff method for determining soil organic matter, and proposed modification of the chromic acid titration method. Soil Sci., 37:29-38, 1934.

YEOMANS, J.C. \& BREMNER, J.M. A rapid and precise method for routine determination of organic carbon in soil. Commun. Soil Sci. Plant. Anal., 19:1467-1476, 1988. 